\title{
Toward Autonomous Navigation of Spacecraft on the Observed Periodic Radiation of Pulsars
}

\author{
A.E.Avramenko and B.Ya.Losovsky \\ P.N.Lebedev Physical Institute of Russian Academy of Sciences, Russia \\ Email: avr@prao.ru
}

\begin{abstract}
The analytical coupling of the Doppler shift of the periodic pulsar radiation with the motion parameters of the observer in any chosen coordinate frame is shown. The motion parameters and the deviation of the spacecraft from the calculated position are associated with the Doppler shift of radiation of the pulsar. According to the coherent radiation of the pulsar in space and time, due to stable rotation parameters, the uniform physical time scales, invariant in any coordinate frame, including on-board, is formed. In the orthogonal coordinate system with axes beginning at the center of mass of the spacecraft and non-rotating axes relative to the barycenter of the Solar system, the projections of the radius vector and spacecraft deflection velocity in the direction of the pulsar are obtained. According to the observed rotation parameters of the pulsar, which are not correlated with its movement, inertial coordinate reference systems are synchronized by the criterion of invariance of analytical pulsar time scales in each of them. As an illustration, the decade data on the timing of the pulsar B0531+21 determined its own position and movement with an estimated accuracy within about $10 \mathrm{~m}$ and $10^{-1}-10^{-2} \mathrm{~m} / \mathrm{s}$, respectively, with the subnanosecond resolution of the measured intervals and corresponded distances.
\end{abstract}

Keywords: spacecraft, position coordinates, velocity vector, pulsar, radiation period, time scales, inertial systems, synchronization.

\section{Introduction}

The solution of many problems of calculating the parameters of the motion of a spacecraft with acceptable accuracy can be obtained if we consider the effect on it of only the most strongly attracting body, and neglect the influence of all other celestial bodies of cosmic ballistics. According to Newton's second law, the differential equation of absolute motion in vector form is expressed as follows [1]:

$$
m \ddot{r}=m \frac{d^{2} r}{d t^{2}}=F_{\Sigma} .
$$

Here $\ddot{r}$ is the full acceleration; $r$ is the radius vector; $r(x, y, z)=\sqrt{x^{2}+y^{2}+z^{2}}$ is the distance from the origin; $t$ is time, independent variable; $F_{\Sigma}$ is the total force acting on the spacecraft.

In this case, we obtain analytical solutions of the equations of motion of a ballistic unperturbed, or Keplerian motion of a spacecraft, which describe it completely. When the spacecraft moves around the Earth, in general, the disturbing force arising due to non-sphericity and the uneven density of the Earth, as well as the aerodynamic force that occurs when spacecraft move in dense layers of the atmosphere, is additionally taken into account.

The content of the navigation problem solved by the radio navigation system is the determination of the space-time coordinates of the spacecraft, as well as its velocity components. In addition to the complete set of linear coordinates relative to the base inertial coordinate system, the expanded consumer state vector should also include the time correction of the time base of the spacecraft relative to the system time scale.

To find the state vector of the spacecraft based on the solution of the navigation problem, a functional relationship is used between the physical values of the delay, the Doppler frequency shift of the radio signal, measured using various combinations of distance measuring methods, and navigation geometrical definitions. The navigation function acts as a generalizing concept of a rather complex algorithm of navigation definitions of a spacecraft using measured radio navigation parameters. 
It should be noted that the most difficult problem is the exact binding of the spacecraft to the system time, for two reasons. First, it is difficult to ensure the long-term stability of the spacecraft's on-board clocks, approaching the parameters of controlled clocks at ground points. And, secondly, it is especially difficult to combine physical time scales in the widely spaced ground-space reference systems that are not synchronized with each other, which are not inertial in general. Thus, the corresponding determination of the coordinate time of a spacecraft is a problem not less, but even more difficult than simply determining a location in space, since the current location of the spacecraft is tied to the exact definition of system time in relativistic coordinate systems of 4-dimensional space-time.

The basis of our approach to solving the problem of autonomous navigation of a spacecraft is the Galilean principle of relativity, according to which all physical processes, phenomena occur equally in inertial reference systems. In such systems, all physical equations are invariant, and their solutions under the same conditions are the same. Since we are not limited in the choice of coordinate reference systems, in addition to observing the specified conditions to be checked, which are also conditions for fulfilling the general physical laws of energy-momentum conservation, angular momentum, the origin of the inertial system can be selected at any point in space, the movement of which is defined in some inertial system, for example, barycentric and geocentric ones.

The choice of pulsars as highly stable sources of periodic radiation, localized at infinity, creates the same conditions for the formation of highly stable time scales, coordinated in any reference system, automatically synchronizing inertial systems, following the principle of simultaneity of the observed events of pulsar radiation at any point of each of these systems.

The target result of this approach is a purely kinematic solution of the navigation mission, obtained only from trajectory measurements, which does not require preliminary knowledge of the disturbing forces. Deviations of the location and velocity of spacecraft from the calculated values are found from the observed deviations of the arrival time of the observed pulses of pulsar radiation due to the Doppler effect detected on-board the spacecraft on a stable pulsar time scale, which is a synchronizing time scale equally distributed by all inertial coordinate systems.

\section{The Physical Basis for Measuring the Motion and Position of Celestial Bodies by Timing the Coherent Periodic Radiation of Pulsars}

Electromagnetic (light and radio) waves can propagate in free space, so that their speed of propagation does not depend on the source motion. If we consider not a steady-state field, but a sudden disturbance, then, in accordance with the law of propagation of the wave front, there is a movement of the surface separating the area already filled with electromagnetic radiation from that area which the disturbance has not yet reached.

If the equation of the moving wave front is

$$
\omega(x, y, z, t)=\text { const }
$$

then the law of propagation of the wave front has the form [2]:

$$
\frac{1}{c^{2}}\left(\frac{d \omega}{d t}\right)^{2}-\left[\left(\frac{d \omega}{d x}\right)^{2}+\left(\frac{d \omega}{d y}\right)^{2}+\left(\frac{d \omega}{d z}\right)^{2}\right]=0 .
$$

For an electromagnetic field, this form of law follows from the Maxwell equation.

Equation (3), which is not related to the specifics of light waves and the electromagnetic field, has a very general character and refers to the extremely rapid propagation of a wave front of any nature with the speed of light in a vacuum, where $\mathrm{c}$ is its value. The law of propagation, expressed by the equation (3), is the main postulate of the physical theory of relativity.

Equation (3) is a mathematical expression of the fact that any point on the wave surface (wave front) moves in the direction normal to the surface at a constant speed equal to the speed of light. This also implies the straightness of the beam, defined as the locus of the indicated points. At the same time, the equation reflects the fact that the space is homogeneous, its geometry is Euclidean. Finally, it contains a statement about the independence of the speed of light on the speed of the source [2].

Here it is important to emphasize the necessary requirement for the location of the source of the electromagnetic field, even more precisely, to its distance from the Solar system, in which all the physical processes we observe occur. To ensure that the curvature of the front surface of the wave is 
$K=1 / R^{2}$, which is inversely proportional to the square of the radius of the sphere centered on the source, does not distort the time on the front of wave at different points in the Galileo space of the Solar system, the distance to the source must be many orders of magnitude exceed the size of the Solar system. Under such conditions, the space in which the front of the pulsar radiation wave propagates has zero curvature, which is inherent in space at infinity, and fully complies with the laws of Euclidean geometry for all physical processes occurring in it, including the physical the process of propagation of the wave front of the periodic radiation of a pulsar.

Thus, the concept of an inertial reference system can now be generalized by including in its definition, in addition to Newton's first law, the law of propagation of a wave front. This also implies the generalized formulation of the Galilean principle of relativity, which is essentially the basis of inertial systems linking three-dimensional Galilean space and time in it, relating to any point in space corresponding to the Lorentz transformations. Since the physical meaning of space is defined at any point, the inclusion of an electromagnetic source, whose wave front reaches any point in the time determined by the Lorentz transformations, is a necessary condition for the coordinate time at any point in the coordinate system while respecting the principle of simultaneity in each of them.

These are the theoretical foundations of the property of homogeneity of four-dimensional space-time, in which all physical processes take place, including the observed movement of space bodies and the movement of the spacecraft.

The dispersion delay of the propagation front of the emitted radio waves $t_{D}$ in the interstellar medium at the frequency $f$ is determined from the known value of the dispersion measure DM as follows:

$$
t_{D}=D M \cdot f^{-2} e^{2} / 2 \pi m_{e} c
$$

The time of arrival the radio signal of the pulsar radiation, which is determined by the group velocity $V_{g}$ of wave propagation in the interstellar medium, differs depending on its frequency $\omega[3]$ :

$$
V_{g}=\left[1-\omega_{p}^{2} / \omega^{2}\right]^{1 / 2} c
$$

here $\omega_{p}=\left(4 \pi n e^{2} / m_{e}\right)^{1 / 2}$ is the plasma frequency of the medium. If follows that pulses observed at higher radio frequencies arrive earlier at the telescope than their lower frequency counterparts.

The difference in the time of arrival of a pulse $\Delta t$ at two frequencies $\omega_{1}$ and $\omega_{2}$, from a pulsar located at a distance $l$ from the observer is given by the following expression:

$$
\Delta t=2 \pi e^{2} n_{e} l\left(\omega_{1}^{-2}-\omega_{2}^{-2}\right) / m_{e} c
$$

After the corresponding frequency-dependent compensation for the lag of the received signal in the entire frequency spectrum, the pulse waveform is restored, indicating the homogeneity of the space in which the wave front propagates at a constant speed.

From the observed dispersion delay $\Delta t$ the magnitude of the dispersion measure $D M$ corresponding to the in-phase propagation of the electromagnetic common-mode wave front over the entire radio frequency spectrum, is determined:

$$
D M=\int n_{e} d l
$$

In expressions (6) and (7) the following notation is used: $e$ is the electron charge, $m_{e}$ is its mass, $n_{e}$ is electron concentration.

As an example, in [4], the spectral structure of the B1356-60 pulsar radiation signal with a rotation period of $0.128 \mathrm{~s}$ received at the radio telescope in the Parkes in the ranges of $0.4 \mathrm{GHz}$ and $1.4 \mathrm{GHz}$. These observations directly confirm the physical homogeneity of the medium along the propagation path of the radiation signal. The wave front, having overcome the distance of about 16 light years in the interstellar medium with the dispersion measure $\mathrm{DM}=295 \mathrm{~cm}^{-6} \mathrm{pc}$, fully preserves the discrete nature of pulsed radiation events with a high degree of temporal resolution of observed radiation events of the order of $1 \mathrm{~ms}$.

The moment of the passage of the front wave of a point source, at a distance of $10^{5}-10^{6}$ astronomical units, is determined only by the local geometrical position of the observer, for which the pulsar with respect to any point of the Solar System is at infinity. In this case, the wave front propagated under identical conditions with constant speed is reaching the observation point at the moment, which is determined only by the spatial coordinates of this point and can be numerically expressed in comparable values at any other point with estimated accuracy. 
Since the velocity of propagation of a pulsar wave front is constant, and the periodic emission of a pulsar, determined by the stable parameters of the pulsar's rotation, is coherent in space and time at infinity and does not depend on the motion of a point source of radiation, solving the navigation task of the spacecraft reduces to measuring the absolute values of the parameters of the motion of a spacecraft relative to pulsars, in which their position in space does not change in time.

According to the coherent radiation of the pulsar, expressed through stable rotation parameters, uniform physical time scales are formed, invariant in any coordinate frame, by which the observed arrival times of radiation waves are measured and the navigation parameters of the spacecraft in the coordinate system are calculated with the origin at the center of mass of the spacecraft. If the measured vector values of the position and speed of the spacecraft do not correspond to the pre-calculated values on the trajectory, then the deviations from the arrival moments of the radiated waves will be used to find the mismatch values with the pre-calculated values for which the spacecraft maneuver can be decided.

Next, turning to the physical problem of measuring the parameters of the motion of a cosmic body in space, we consider it by the example of the observed Doppler-effect induced by the spontaneous motion of a pulsar uncorrelated with its coherent periodic radiation. In this case, it will be necessary, first, to show the identity of the navigation parameters of the space object motion measured in a topocentric observational coordinate system with the origin in the phase center of the radio telescope and in the inertial barycentric coordinate system, in which the topocentric observational data are reduced using ephemeris coordinate transformations. Secondly, it is necessary to verify the numerical equivalence of the parameters of the spontaneous motion caused by the Doppler-effect, measured from the observed coherent periodic radiation of the pulsar in these coordinate systems. And, thirdly, we have to make sure that the coordinate systems synchronized by the periodic radiation of the pulsar are inertial, inertial systems, in any of which all measured physical quantities for calculating the navigation states of a space object are completely determined by kinematic equations, the parameters of which are the rotation parameters of pulsar.

The measurements made on-board of the deviations of the motion parameters from their calculated values, while observing the listed conditions, are the basis for calculating and subsequently performing a controlled corrective maneuver of the spacecraft at any chosen point of the trajectory (orbit) with a priori estimated accuracy.

\section{Detection of Motion Parameters in the Observed Periodic Radiation of the Pulsar}

There is a relatively small class of pulsars, all within a few dozen stars belonging to supernova remnants, for which one of the defining features is their movement under the influence of a shock wave in the medium of a gaseous envelope after a supernova explosion [5]. The extremely high dynamics of processes observed in galactic nebulae with a central neutron star formed after a supernova explosion confirms the accelerated expansion of the gaseous envelope under the influence of energy emanating from the central star and causing shock waves in an ionized circumstellar plasma observed in a wide frequency range. So, after the explosion of the supernova SN 1054, registered only about $10^{3}$ years ago, a new neutron star appeared in the Crab Nebula, now identified with the pulsar B0531+21 [6]. The star is in the constellation Taurus at a distance of about 6500 light years (2 kpc) from Earth.

The observed pulsed periodic radiation of pulsars is closely related to the fundamental physical properties of neutron stars. Our analytical model of timing allowed us to find such fixed values of the rotation period and derivatives (for millisecond pulsars, only the first derivative is significant) for the measured moments of selectively observed pulsar radiation events for the initial epoch of observations for which the divergence of the observed and calculated by the fixed value of the period and time derivatives the arrival of the observed radiation pulses approaches near zero value [7].

For several decades, the BSA LPI (Pushchino) radio telescope has been regularly timing the B0531+21 pulsar, along with a group of other pulsars, among them B0809+74, B1919+21, B0834+06, $\mathrm{J} 1509+5531$, B2217+47, B0329+54. For each of them, we obtained the agreed observable rotation parameters $P_{0}^{*}, \dot{P}, \ddot{P}$. 
The identified stable patterns of the observed period of radiation and derivatives in the process of gradual slowing down of rotation, indicating the coherence of periodic radiation of pulsars, almost completely eliminated the effect of unmodeled timing noise, which allowed direct detection to determine the exact value of the second derivative, consistent with other rotation parameters, and this in turn led the deceleration rate for all observed pulsars to the numerical invariant $\mathrm{n}=-(0.9 \pm 0.2)$, corresponding to coherent radiation of a pulsar [8].

As a result, an analytical model of the observed radiation intervals of the pulsar, not affected by the random noise of timing, made it possible to proceed to the direct detection of the physical effects associated with the observed coherent radiation of the pulsars.

In the analytical timing model, the observed rotation parameters $P_{0}^{*}, \dot{P}, \ddot{P}$ are found from the equations of the periodic pulsar radiation intervals observed with the radio telescope associated with the ephemeris of the celestial bodies of the Solar system:

$$
P T_{i}=\left(1+\alpha_{i}\right)\left(P_{0}^{*} N+\frac{1}{2} P_{0}^{*} \dot{P} N^{2}+\frac{1}{6}\left(P_{0}^{* 2} \ddot{P}-2 P_{0}^{*} \dot{P}^{2}\right) N^{3}\right)_{i}
$$

Here $P T_{i}$ are the numerical values of the intervals observed with the radio telescope; $\alpha_{i}$ is the relative deviation of observed intervals $P T_{i}$ from $P T_{\text {calc }}$, calculated from the observed rotation parameters $P^{*}, \dot{P}, \ddot{P}$

Thus, we have the following relations:

$$
P T_{\text {obs }}=P T_{i} ; \quad P T_{\text {calc }}=\left(P_{0}^{*} N+\frac{1}{2} P_{0}^{*} \dot{P} N^{2}+\frac{1}{6}\left(P_{0}^{* 2} \ddot{P}-2 P_{0}^{*} \dot{P}^{2}\right) N^{3}\right)_{i}
$$

From expressions (3.1) and (3.2) follows:

$$
\alpha_{i}=\frac{P T_{o b s}}{P T_{\text {calc }}}-1
$$

For the absolute majority of pulsars, the value of the coefficient $\alpha_{i}$ does not exceed of $10^{-15}-10^{-16}$ in any interval of observations, and, therefore, when going for the infinite duration of observations, this value in the limit tends to zero:

$$
\lim \frac{P T_{o b s}-P T_{c a l c}}{P T_{\text {calc }}}=0, \text { if } N \rightarrow \pm \infty
$$

The upper value of this limit is determined by random timing noise, the level of which does not affect the result of the parametric approximation of the observed intervals of the coherent radiation source, which corresponds to their convergence, as, for example, for the pulsars B1919+21 and B0809+74 [8,9].

The numerical value of period obtained from equation (3.1) is a function of the time argument, measured from the initial observed radiation event $(\mathrm{N}=0)$ :

$$
P(t)=P_{0}^{*} \pm\left(\dot{P} \cdot t+\frac{1}{2} \ddot{P} \cdot t^{2}\right) ; t=P_{0}^{*} N ;-\infty<N<\infty .
$$

Note the symmetric dependence of the period, both with increasing and decreasing time argument, which is confirmed by archives of observational data, pulsar catalogs and directly indicates the coherence of the periodic radiation of the pulsar, the characteristic feature of which is the consistency of the predicted and retrospective radiation parameters corresponding to stable parameters of pulsar rotation unlimited duration of the observations.

The observations on radio telescope BSA LPI of pulsar B0531+21, unlike other pulsars of observed group, detect an anomalous deviation the approximated period $\mathrm{P}$ and intervals $\mathrm{PT}$ that do not fit into a convergent parameterized power series of the observed PT intervals. However, the observed rotation parameters obtained from the equation (8), were matched with the criteria of coherence and correspond to the typical monotone spin down braking index within $\mathrm{n}=-(0.9 \pm 0.2)$, that is, they were in the same range as other pulsars of the group.

Meanwhile, the relative magnitude of the observed deviations of the period $\Delta P$ during the two-year observations reached the order of $10^{-9}$, which is $5-7$ orders of magnitude greater than the other pulsars of the observed group. In addition, about, not later than 21.02.2012 (MJD 55978), it has inverted the sign of the deviation of $\mathrm{P}$, and further observation showed a sharp increase in its order of magnitude up to $10^{-6}[8]$. The absolute value of the period deviations is expressed as follows: 


$$
\Delta P(t)=\alpha(t)\left[P_{0}^{*} \pm\left(\dot{P} \cdot t+\frac{1}{2} \ddot{P} \cdot t^{2}\right)\right]
$$

These spontaneous deviations of the observed emission period that is not associated with changes in the pulsar rotation parameters, can be interpreted as a Doppler shift due to the motion of the pulsar because of the effects of unbalanced forces in radial directions within the full angle of rotation of the pulsar around the axis of rotation.

Expression (13) can be written in discrete form, which corresponds to the period deviations for the fixed moments of the i-th observed radiation event:

$$
\Delta P_{i}=\alpha_{i}\left[P_{0}^{*} \pm\left(\dot{P} \cdot t+\frac{1}{2} \ddot{P} \cdot t^{2}\right)\right] ; t=P_{0}^{*} N_{i} .
$$

Similary expressed observable deviations of intervals in the region from the initial event to the $i$-th observed event:

$$
\Delta P T_{i}=\alpha_{i} \cdot P T_{i}=\frac{1}{P_{0}^{*}} \int_{0}^{t_{i}} \Delta P(t) d t .
$$

The offset of the intervals in the fixed region between the observed events $i, j(j<i)$ is calculated by the following relationship:

$$
\Delta P T_{j, i}=\frac{1}{P_{0}^{*}} \int_{t_{j}}^{t_{i}} \Delta P(t) d t
$$

The radial velocity of the movement of the pulsar along the line of sight is defined on an epoch of observation as follows:

$$
V_{i}=\alpha_{i} \cdot c
$$

Here $c$ is light speed. Displacement $\Delta L_{i . i}$ of a pulsar over the time between randomly chosen observed events in an interval $\left\{P T_{j}, P T_{i}\right\}$ is calculated on the follow ratio:

$$
\Delta L_{j, i}=V_{i} \cdot \Delta P T_{j, i}=\alpha_{i} \cdot c \cdot \Delta P T_{j, i}
$$

Replacing continuous function with its discrete approximation on the equation (8), and assuming that the change of the observed period in an interval $\{\mathrm{ti}, \mathrm{ti}\}$ happens evenly, we obtain deviations of observed intervals in the form of the final differences between any two observed events of radiation of a pulsar within this interval:

$$
\Delta P T_{j, i}=\frac{\alpha_{i}+\alpha_{j}}{2}\left(P T_{i}-P T_{j}\right)
$$

Thus, all the necessary expressions have been obtained that relate the parameters of motion and the position of the pulsar to the observed rotation parameters, which, in turn, are obtained analytically by approximating the observational data of timing. Using these relationships, we can determine the kinematic characteristics of a space object associated with the observed Doppler effect of periodic radiation of a pulsar, detected by stable rotation parameters of a monotonously slowing pulsar not correlated with its movement parameters.

\section{To the On-Board Measurement of Navigation Status Spacecraft}

The movement of the pulsar, detected by changes in the radiation period observed with the radio telescope, allows us to extend the results of measurements of the parameters of the movement of the pulsar relative to the observer or, equivalently, the movement of the observer relative to the pulsar, to any coordinate system, including the moving coordinate system, with its origin coinciding with the center of mass of the spacecraft.

If we assume that a still, undisturbed pulsar is timed on-board a moving spacecraft, two possible cases can be considered. In the first of them, the motion of a spacecraft is considered a priori known, it corresponds exactly to the equation of its trajectory (ephemeris), and so we have a similar picture and the same results with the observation of a pulsar on a ground-based telescope, as, for example, in case of pulsars B0809+74, B1919+21, in which there are no observed signs of movement. Or, in the second case, 
if the motion of the spacecraft occurs deviations from the estimated trajectory, we obtain the results of measuring the deviations of the position and velocity in the direction of the timed pulsar, similarly to the case discussed above in Section 3 for the pulsar B0531+21.

To measure the motion parameters of a spacecraft, we cannot do enough to limit ourselves to choosing only one coordinate system, for example, the inertial barycentric one, the origin of which coincides with the fixed center of mass of the Solar System, because in real conditions the pulsars used to measure navigation status of the spacecraft, they are observed with both on ground-based and onboard spacecraft telescopes. In any case, no matter in which coordinate system the pulsars are observed, the parameters of the motion of the spacecraft measured from these observations, in the physical sense, should not depend on the choice of the coordinate system for monitoring the pulsars.

Practical implementation of the coordinate systems in astronomy is attained by setting the coordinate values of the reference celestial objects. Thus, the coordinate system as a mathematical construct is transformed into meaningful astronomical reference frames (RF). The modern positional astronomy holds a permanent work to maintain the International Celestial Reference Frame (ICRF) and the International Terrestrial Reference Frame (ITRF). The ICRF is defined by the provisions of the quasars in the International Celestial Reference System (ICRS), which is a particular barycentric RF. The ITRF is defined by the provisions of ground reference stations in the International Terrestrial Reference System (ITRS), which is a rotating with the Earth geocentric RF. The relationship of these systems, on the one hand, is given theoretically by solutions relativistic equations of motion the celestial bodies and the Earth's rotation, and on the other hand, is determined from observations [10].

Reference coordinates systems are built so that they adhered to the principle of physical equivalence. Mathematically, it means that in such systems, all physical processes and phenomena occur equally and physical equations have the same form, that is, all reference systems should be equal, and observed them in the same conditions of physical phenomena and processes are indistinguishable. Such reference systems are inertial.

In contrast to the inertial coordinate Newtonian mechanics, the coordinates for the finite (not infinitesimal) space of events have no physical meaning and cannot be directly compared with observational data. Therefore, the problem of comparing of the measured and calculated values becomes methodologically important and physically essential task. In that case, the basis of the choice of coordinate methods for constructing theories of the motion of celestial bodies puts the mathematical approach, taking into account the convenience of the various coordinates for purely mathematical solving of dynamic task, adopted, for example, in the calculation of the coordinates in the equations of planetary ephemeris of the Solar System [11]. The subsequent transition from ephemeris coordinates to the coordinate-independent physically measured values is achieved by combination of solutions of the dynamic task (the motion of bodies) and the kinematic task (propagation of light) in the same coordinates. To comply with the principle of equivalence is only important that both tasks have to be solved in the same coordinates [12].

A special place in the hierarchy of space coordinate systems takes topocentric coordinate system, the origin of which, unlike the geocentric system, can be selected at any observation point on Earth's surface, approximated as the geoid, which is associated with the system of astronomical coordinates, or ellipsoid, connects with the system of geodetic coordinates. On average, both approximations are almost identical, but in some areas of the surface differences can be substantial. Therefore geodetic methods on the surface built a network of local reference ellipsoids, which are tied to the topocentric coordinate reference system according to the average terrestrial ellipsoid. The mutual position of the observation points is determined from these coordinates.

The fourth coordinate of a relative reference frame is a coordinate time scale, which is the mathematical argument in the appropriate theories of the motion or rotation of celestial bodies in the Solar system. It is not an observable and directly measurable quantity that can be directly compared with the calculated values. In astronomical calculations used the ephemeris Earth's time TT (Time Terrestrial), which is the argument of the time of geocentric ephemerides. Ephemeris time TT, is reduced to the ephemeris scale of the TDB, which is the argument of heliocentric ephemeris, whose unit is also the SI second of the TAI atomic time.

The equation of the intervals of coherent radiation of pulsar (8), which is forminvariant in the both barycentric and topocentric frames, determines the physical time in these coordinate systems in the form of an analytic function of the observed period and its derivatives. As numerical values of the observed 
period for coinciding local time epochs in any coordinate system are identical, therefore, in general, on the observed divergence of local coordinate time $t_{T R S}-t_{B R S}$ such value of the rotation period is defined which corresponds to the rotation parameters, measured at the epochs of observation in these coordinates. So in each inertial reference frame, there is its proper physical time - the changed local time of Lorentz. The physical time obtained in this way in inertial systems corresponds to the implementation of the principle of simultaneity of the observed events and, as a consequence, automatically - the synchronization conditions of the coordinate systems from the observed periodic radio emission of the pulsar. The amendment in the coordinate values leads only to a change in the relationship of the physical time with the coordinate values. In each inertial system in Galilean coordinates there exists a single physical time $t$, whose lines are orthogonal to the three-dimensional space.

Figure 1 shows the characteristics of the movement of the pulsar B0531+21, measured in topocentric and barycentric coordinate systems, according to the timing on the radio telescope BSA LPI in the frequency range $111 \mathrm{MHz}$ within the interval 01.2010-06.2018. The observations of pulsar $\mathrm{B} 0531+21$ detect an anomalous deviation of the coefficient $\alpha_{i}$ in the equation (8), expressing the relative deviation of observed intervals $P T_{i}$ from $P T_{\text {calc }}$, calculated from the observed rotation parameters $P_{0}^{*}, \dot{P}, \ddot{P}$. However, the observed rotation parameters obtained from the equation (2), matched with the criteria of coherence and correspond to the typical monotone spin down with braking index $\mathrm{n}=-(0.9 \pm 0.2)$. Meanwhile, the relative magnitude of the observed deviations of the period $\mathrm{P}$ during the two-year observations reached the order of $10^{-9}$, which is $5-7$ orders of magnitude greater than the other pulsars, as B1919+21 and B0809+74. In addition, 21.02.2012 (MJD 55978), it has inverted the sign of the deviation of the observed $\mathrm{P}$, and further observation showed a sharp increase in its order of magnitude up to $10^{-6}[8,9]$.

Parameters of the movement-the speed and movement of a pulsar, as well as rotation parameters, are defined by the Equation (2). But, for PSR B0531+21, it is necessary to consider that the dimensionless parameter $\alpha_{i}$ of approximation of intervals (2) is associated with the movement of a pulsar here and is interpreted as the Doppler shift of the observed period of radiation owing, in accordance with the relation (14), to the movement of a pulsar under the influence of unbalanced forces in the radial directions. The radial velocity and displacement of the pulsar along the line of sight is defined on ratios (17) and (18), respectively.

Over the span of nearly 6-year observations of pulsar B0531+21 within 2010-2015, its velocity changed in range, from $0.1 \mathrm{~m} / \mathrm{s}$ or less up to about $500 \mathrm{~m} / \mathrm{s}$, displacement of pulsar along the line of sight changed from a few hundred meters up to about 100 million $\mathrm{km}$ [8]. With further expansion observations of pulsar B0531+21 more than 8-year within 2010-2018, the speed of movement has increased up to about $4000 \mathrm{~m} / \mathrm{s}$, displacement of pulsar exceeded $10^{9} \mathrm{~km}$ [9]. This unprecedentedly wide dynamic range of measured values of velocity $\left(10^{4}-10^{5}\right)$ and displacement $\left(10^{8}-10^{9}\right)$ of the pulsar is due to the extremely accurate, with subnanosecond resolution $\left(10^{-9}-10^{-11}\right) \mathrm{s}$ of the observed radiation intervals approximated in terms of the rotation parameters of the pulsar.

As follows from a comparison of the measured values of the velocities and displacements of the pulsar in graphs Figure 1c, their numerical values in the topocentric and barycentric coordinate systems are visually the same in both systems. It should only be noted here that when the direction of movement of the pulsar is reversed to the opposite, what happened near (no later) MJD 55978, certain problems arose with the fitting of barycentric intervals performed by the TIMAPR timing program [13]. Excess components in the millisecond range were superimposed on the regular barycentric TOAs, the consequences of which appeared in the interval of about one year after this jump, traces of their presence can be seen by comparing the graphs Figure 1c (left and rights). This feature of the coordinate transformation program, however, did not affect the invariance of the observed movement and the identity of its estimated parameters in the coordinate systems as a whole.

In this way, according to the observations of the pulsar B0531+21, the full similarity of its movement observed in the topocentric and barycentric coordinates as well as of their periodic radiation, is shown. Thus, in other words, it is shown that coordinate systems synchronized by the coherent radiation of a pulsar are inertial. In these systems, both physical processes - radiation and the movement of a pulsar are expressed by the invariant equations of the observed radiation intervals, the solutions of which, in accordance with the principle of simultaneity, are coincident rotation parameters in each of them. 
The same ratios of the intervals and observed rotation parameters will be performed on the moving spacecraft along the trajectory, with the only difference that, unlike fixed points that set the origin of topocentric systems in coordinates on the Earth's surface, can move relative to the calculated motion trajectory. In this case, according to the deviations of the observed period of rotation of the pulsars, navigation parameters are estimated - the displacement and the velocity of the spacecraft in the direction of the pulsars, which form an orthogonal (in principle) coordinate reference system with nonrotating axes relative to the reference system ICRS.

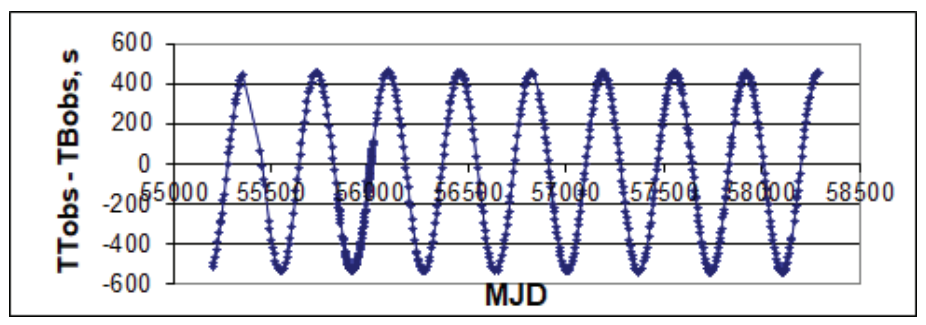

1a. Difference of topocentric (TTobs) and barycentric (TBobs) Times of Arrival (ToAs) due to the annual circulation of the Earth around the Sun
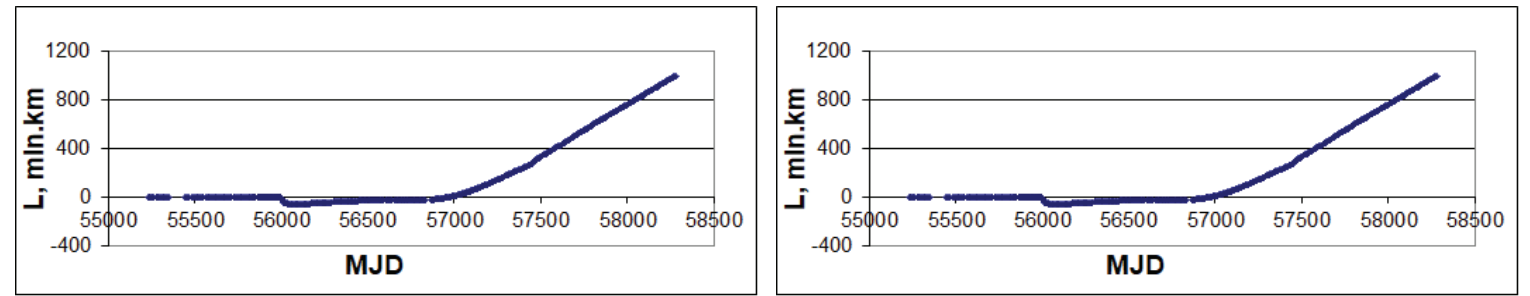

1b. Observed displacement L of the pulsar B0531+21 calculated according to the ratio (18) in topocenter (left) and barycenter (right). Rotation parameters: $P=0.033403347409400 \mathrm{~s}(48743.0), \dot{P}=4.2096 \cdot 10^{-13}$ correspond to their values specified in the Catalogue [14]. The rotation period $P_{0}(T T)$ (topocenter) and $P_{0}(T B)$ (barycenter) at the initial epoch MJD found from the approximation of intervals TTobs and TBobs, respectively, are followed: $P o(T T)=0,0336388778584014 \mathrm{~s}$ (MJD 55218.787); Po(TB)=0,0336388780596485s (MJD 55218.792).
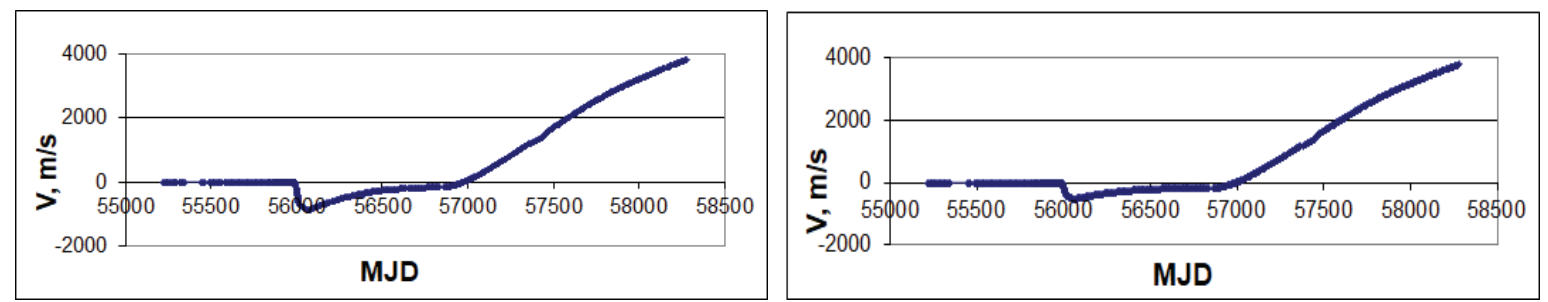

1c. Observed velocity $\mathrm{V}$ of the pulsar B0531+21 in topocenter (left) and barycenter (right). Changes of velocity $\mathrm{V}$ calculated according to the ratios (14) and (17), which are unpredictable, have signed-variable character and vary in range from $-500 \mathrm{~m} / \mathrm{s}$ up to $3000 \mathrm{~m} / \mathrm{s}$ and more.

Figure 1. Movement of the pulsar B0531+21 observed on the radio telescope BSA LPI (Pushchino).

By spreading the parametric pulsar time scales to the observational coordinate systems, by synchronizing the periodic radiation of the pulsar, they are converted into inertial systems, in each of which the simultaneity principle is fulfilled at all points in space, there is a kinematic solution of the physical equations of navigation parameters functionally related to the desired parameters motion indistinguishable in any inertial system. The synchronization of the coordinate systems of reference for the periodic emission of pulsars removes, in particular, the problem of the long-term stability of the onboard time scales inherent in the commonly used aboard spacecraft atomic scales.

The same conditions for measuring the motion parameters and the position of a spacecraft using the same numerical parameters for pulsar rotation in any coordinate system - barycentric, topocentric, and on-board spacecraft - ensure the consistency of measured values with an accuracy determined by the 
scales of coordinate time. Since these scales are determined by the agreed parameters of the rotation of the pulsar, which are the same for the coinciding epochs of the coordinate time, the inaccuracy of the measured parameters of the spacecraft, which depends only on the difference in the coordinate time scales, does not go beyond a few nanosecond values at a subnanosecond resolution of the observed intervals and distances.

Thus, synchronization of the barycentric, topocentric and on-board inertial coordinate systems, based on the observed periodic radiation of the pulsar, is completely determined by the numerical values of the rotation period at the epoch of the initial radiation event and derivatives, which determine the coordinate time scales agreed by the criterion of simultaneity of the observed events at any point in space of each coordinate system within any length of observation.

\section{Prospects for the On-Board Timing of Pulsars as Sources of High- Energy Radiation}

Along with the considered capabilities of radio pulsars to determine the navigation parameters of spacecraft, attention has recently been drawn to the use of X-ray. The advantage of using X-ray signals over radio waves is that X-ray telescopes can be smaller and lighter. Navigation based on periodic X-ray radiation from pulsars attracted the attention as an opportunity to determine the coordinates of the spacecraft without communication with the Earth, when exploring remote areas of space, both in the Solar System and beyond.

On June 5, 2017, the NASA SEXTANT Device (Station Explorer for X-ray Timing and Navigation Technology), developed by the Goddard Space Flight Center, was delivered to the International Space Station (ISS), designed to test XNAV (X-ray pulsar-based navigation and timing) on orbit with the NICER project (Neutron star Interior Composition Explorer) [15].

The primary goal of the software application is to demonstrate, for the first time, the determination of the navigation solution for a spacecraft (in this case, the ISS) based solely on X-ray pulsar measurements onboard and in real. The pulsar template approximates the stable waveform that would be obtained in an extremely long timing on-board similar to the radio pulsar time.

Because time is referenced to GPS, for SEXTANT the pulsar measurements are used only for determining the position and velocity of the ISS. A future pulsar navigation system should be independent of GPS. This will require a stable local clock onboard, which the pulsar measurements would then steer to a pulsar-based time standard. This will require more pulsars providing measurements to account for the additional dimension of the problem (a minimum 4 pulsars, rather than 3$)$.

The timing capabilities of pulsars to determine on-board navigation parameters can potentially be enhanced by a highly energetic gamma-ray. The radio pulsars found as the pulsing gamma-ray sources by the Large Area Telescope (LAT) on the board of the Fermi Gamma-Ray Space Telescope. In article [16] the correlation between gamma-ray luminosities and radio luminosities is found. It allows one to select those objects from all set of the known radio pulsars that can be detected as gamma pulsars with the high probability. The authors give a list that contains more than 100 potential gamma pulsars. On the other hand, the known catalogue of gamma pulsars contains some sources which are not known as radio pulsars at this moment. Some of them have the large values of gamma luminosities and according to the obtained correlation, it can be expect marked radio emission from these objects. The list of such pulsars end expected flux densities to search for radiation at frequencies 1400 and $111 \mathrm{MHz}$, is given.

Using the periodic radio- and gamma- radiation, determined by the agreed parameters of the rotation of the pulsar, the position and velocity of the moving spacecraft within any time interval are measured both by ground observation means and on-board the spacecraft under the same conditions [17]. The results of navigation measurements are numerically compatible in any coordinate system, each of which is synchronized with the observed coherent radiation of the pulsar.

Due to the identity synchronizing pulsar time scales in any coordinate system, it is possible, for example, to pre-test the on-board X-ray or gamma-ray scales by comparing them with the timing data of radio pulsars on ground-based radio telescopes, followed by setting the rotation parameters consistent with the calculated position of the on-board spacecraft for the corresponding initial epoch. 


\section{Conclusion}

The target result of this approach is a purely kinematic solution of the navigation mission, obtained only from trajectory measurements, which does not require preliminary knowledge of the disturbing forces. Deviations of the location and velocity of spacecraft from the calculated values are found from the observed deviations of the arrival time of the observed pulses of pulsar radiation due to the Doppler effect detected on-board the spacecraft on a stable pulsar time scale, which is a synchronizing time scale equally distributed by all inertial coordinate systems.

By spreading the parametric pulsar time scales to the observational coordinate systems, by synchronizing the periodic radiation of the pulsar, they are converted into inertial systems, in each of which the simultaneity principle is fulfilled at all points in space, there is a kinematic solution of the physical equations of navigation parameters functionally related to the desired parameters motion indistinguishable in any inertial system. The synchronization of the coordinate systems of reference for the periodic emission of pulsars removes, in particular, the problem of the long-term stability of the onboard time scales inherent in the commonly used aboard spacecraft atomic scales.

\section{References}

1. N.M. Ivanov and L.N. Lysenko, Ballistics and Navigation Spacecraft, (2 ${ }^{\text {nd }}$ edition). Drofa, Moscow, 2004 (in Russian).

2. V.A. Fok, Einstein's Theory and Physical Relativity, (2 ${ }^{\text {nd }}$ edition). URSS, Moscow, 2009 (in Russian).

3. I.F. Malov, Radio Pulsars. Nauka, Moscow, 2004 (in Russian).

4. D.R. Lorimer and M. Kramer, Handbook of Pulsar Astronomy. Cambridge University Press, 2005.

5. V.M. Kaspi, Neutron Star/Supernova Remnant Associations, 1998. http//arxiv.org/pdf/astro-ph/9803026.pdf

6. F.G. Smith, Pulsars. Cambridge University Press, 1977.

7. A.E. Avramenko, "The Observed Rotation Period as an Identifier of the Pulsar Time Properties", in Pulsars: Theory, Categories and Applications, Nova Publishers, NY, pp.61-72, 2010.

8. A.E. Avramenko and B.Ya. Losovsky, "A Continuous Observing Rotational Stability of Pulsars", in Proceedings of Russian Astrometric Conference, S-Petersburg, 2016, pp. 11-16 (in Russian).

9. A.E. Avramenko, B.Ya. Losovsky, V.D. Pugachev, and T.V.Shabanova, "Compatibility of the Observed Rotation Parameters of Radio Pulsars on Long Time Scales", International Journal of Astronomy and Astrophysics, vol. 8, no. 1, pp.24-45, 2018.

10. V.K. Abalakin, Basics of Ephemeris Astronomy. Nauka, Moscow, 1979 (in Russian).

11. E.V. Pitjeva, "The IAA RAS Fundamental Ephemerides of Planets and the Moon (EPM): their Model, Parameters, Accuracy", Proceedings of IAA RAS, vol. 26, pp. 54-64, 2012 (in Russian).

12. V.A.Brumberg, "Celestial Mechanics: Past, Present, Future", Solar System Research, vol.47, no. 5, pp.347- 358.

13. O.V. Doroshenko and S.M.Kopeikin, "High Precision Pulse Timing for Single Pulsars", The Astronomical Journal, vol.67, pp.986-992.

14. J.H. Taylor, R.N. Manchester, and A.G.Lyne, "Cataloque of 558 Pulsars", Astrophysical Journal Supplement series, vol.88, pp.529-568.

15. P.S. Ray, K.S. Wood, and M.T. Wolff, "Characterization of Pulsar Sources for X-ray Navigation", in arXiv:1711.0850\%1 [astro-ph.IM], 2017.

16. I.F. Malov and M.A. Timirkeeva, "Radio Pulsars with Expected Gamma Radiation and Gamma Pulsars as Pulsating Radio Emitters", in arXiv:1712.06990v1 [astro-ph.HE], 2017.

17. A.E. Avramenko, "A Method of Navigation a Spacecraft by Means of Celestial Sources of Periodic Radiation", The Patent of the Russian Federation no. 2453 813, vol.17, 2012. 